\title{
基于民族图案基元可拓语义的产品设计方法
}

\author{
秦臻, 季铁, 刘芳, 刘永红 \\ (湖南大学设计艺术学院 长沙 410082) \\ (sang2@163.com)
}

\begin{abstract}
摘 要：针对在面向民族图案的产品设计过程中，设计者容易忽略民族图案所隐含的复杂语义，导致所设计的产品 脱离图案原本蕴含的文化内涵的问题，提出基于民族图案基元可拓语义的产品设计方法. 利用可拓理论对民族图案 基元的语义进行提取、量化分析、图形解析，以优化产品设计过程中民族图案的运用. 以侗族织锦为例，首先，由当 地文化持有者和设计人员共同对侗族织锦图案基元及其语义进行提取和选择; 然后，对图案基元的语义进行分析， 得出最大可拓设计区间的特征词汇，再对特征词汇进行图解，并分析出最大可拓设计区间的图解；最后，对最大可 拓设计区间的图解进行分析，并以此指导设计实践. 邀请设计师和当地文化持有者共同对设计方案的文化表现性、创 新性及美观度进行优度评估，遴选优度较高的设计方案，设计制作出具体的产品，提升在产品设计过程中对民族图 案运用的准确度与优度.
\end{abstract}

关键词：民族图案；图案基元；可拓语义；产品设计

中图法分类号: TP391.41～DOI: 10.3724/SP.J.1089.2021.18755

\section{The Method of Product Design Based on Extension Semantics of National Pattern Primitives}

Qin Zhen, Ji Tie, Liu Fang, and Liu Yonghong

(School of Design, Hunan University, Changsha 410082)

\begin{abstract}
In the process of product design for national patterns, designers tend to ignore the complex semantics implied by ethnic patterns, which leads to the design of products separated from the original cultural connotation of patterns. In view of this problem, the method of product design based on extension semantics of national pattern primitives is proposed. By using the extension theory to extract, quantitatively analyse and illustrate the semantics of ethnic pattern primitives, the application of national pattern in the product design process is optimized. Taking the brocade of Dong nationality as an example, firstly, the pattern primitives of Dong brocade are extracted and selected by the local culture holders and designers. Secondly, the extension semantics of pattern primitives is analyzed, and the characteristic words of the maximum extension design interval are obtained. Thirdly, the characteristic words are illustrated, and the illustration with the maximum extension design interval is analyzed. Fourthly, the illustration is analyzed to guide the design practice. Finally, the designer and the local culture holders are invited to jointly evaluate the cultural expression, innovation and beauty of the design scheme, select the design scheme with higher quality, design and produce the specific product, all of which improves the accuracy and superiority of the use of national patterns in the process of product design.
\end{abstract}

收稿日期：2020-11-03; 修回日期：2021-04-30. 基金项目：国家重点研发计划(2019YFB1405702). 秦臻(1988一), 女, 博士研究 生, 讲师, 主要研究方向为纺织品设计; 季铁(1972一), 男, 博士, 教授, 博士生导师, 主要研究方向为设计与社会创新; 刘芳 (1976-), 女, 博士, 教授, 博士生导师, 主要研究方向为边缘计算、大数据处理与智能工业设计; 刘永红(1972一), 男, 博士, 教 授, 博士生导师, 主要研究方向为工业设计. 
Key words: national pattern; pattern primitives; extension semantics; product design

民族图案由于其独特的视觉形态和文化内涵, 常被运用于产品设计中. 在产品设计过程中, 如果 只基于视觉效果对民族图案进行提取、运用，容易 造成产品的去语境化及歪曲 ${ }^{[1]}$, 无法展现其文化内 涵. 然而, 对承载文化内涵的图案语义的提取和运 用具有复杂性：一是由于图案的语义根植于当地 的生活、习俗、信仰之中，一个图案往往具有多重 的语义, 而这些语义的权重比例难以描述, 是当地 文化持有者具身化的隐性知识; 二是所运用的图 案的语义也需要符合当今产品设计的需求. 基于 此，本文以可拓语义的方法，由当地文化持有者和 产品设计师共同对民族图案的语义进行提取、分析 和运用，进行基于民族图案的产品设计.

\section{1 理论描述}

\section{1 民族图案基元}

民族图案基元 ${ }^{[2-4]}$ 是指在某一民族的生产生活 中出现的、呈现该民族文化特色的、单个的、典型 的、具有独立语义和稳定形态的图案单元.

一方面，图案基元作为民族图案的视觉和语 义核心被运用于现代产品设计. Saito 等 ${ }^{[5]}$ 利用日本 传统漆器上的云纹图案基元，设计制作了带有近 场通信天线的交互式钥匙扣和午餐盒; Qian 等 ${ }^{[6]}$ 以 柯尔克孜族纺织图案为基础建立民族图案基元库, 作为民族产品设计的基础; Huang 等 ${ }^{[7]}$ 对陶瓷图案 基元的构成形式进行分析，并构建相应的数据库； 董馥伊 ${ }^{8}{ }^{8}$ 对传统栽线毯典型性图案基元进行提取, 以此进行产品设计; 乌云 ${ }^{[3]}$ 基于新疆民族服饰的 图案基元构建图案基元数据库, 设计当地干果的 包装、丝巾等旅游纪念产品：但上述工作多集中于 对民族图案基元视觉形态的提取和运用, 少有涉 及图案基元的语义.

另一方面，图案基元也是计算机技术和设计 学跨学科研究的关注焦点. Jimoh 等 ${ }^{[9]}$ 利用元胞自 动机边缘检测技术识别尼日利亚的手工刺绣图案， 并构建相应的数据库; Sugita 等 ${ }^{[10]}$ 利用视觉模式图 形编码技术对日本传统手工艺中的图案进行识别; Siregar 等 ${ }^{[11]}$ 基于纹理特征提取桑巴传统织物 Kain Lunggi 的图案基元，以保护和推广该项传统工艺; Rangkuti 等 ${ }^{[12-13]}$ 提出基于内容的蜡染图案的识别 与检索方法, 并利用 treeval 和 treefit 决策树函数分
类优化其检索方法; 贾小军等 ${ }^{[14-15]}$ 基于 VGGNet 卷 积神经网络分别对蓝印花布的纹样和纹样基元进行 分类实验, 以识别图案基元, 启发图案设计; 刘菲 朵 ${ }^{[4]}$ 提出民族图案基元分割的基于图的超像素分割 (superpixel graph-based)算法，以有效地提取民族文 化基因，驱动民族产品设计; 何旋 ${ }^{[16]}$ 利用计算机图 像处理技术对印花织物的图案基元进行分割, 提高 检索和设计的效率: 这些工作大多也是从图案基元 的视觉方向出发探讨图案基元提取和运用的方法.

然而，民族图案的价值不仅仅是基于视觉的， 特别是在我国图必有意的文化背景下，民族图案 基元的研究与运用还需要考虑其背后丰富的图案 语义 ${ }^{[17]}$. 尤其是在少数民族地区，有些民族没有 文字，图案是其记录、传承、表达的工具，这些图 案基元根植于当地的传说、习俗、信仰之中，如同 少数民族文化中的一个个故事, 表达着复杂的语 义，对其不加理解地运用会造成文化语义的消解， 所设计出的产品也不具有相应的文化价值. 比如, 侗族织锦中的风鸟纹来源于侗族南迁时曾受神鸟 指引方向的故事, 在侗锦中的风鸟纹通常 4 个相对 出现，寓意八方报喜; 同时它又是尊贵女性的象 征, 常与龙纹结合运用, 成对出现在婚庆用品之 中. 所以，当地文化持有者认为风鸟纹是尊贵、神 秘、灵动、美丽和圆满的象征综合体. 但是, 在这 些象征意义中，具体哪个象征意义所占的权重最 大, 如何运用这些象征意义, 是当地文化持有者具 身化的隐性知识 ${ }^{[18]}$. 而对这样的隐性知识的识别成 为设计中的问题，因为传统的感性词汇表达容易造 成语义编码或解码的错误 ${ }^{[19]}$. 所以, 不仅需要先提 取图案基元的复杂语义, 还需要对这些语义进行理 性的、量化的分析，以有效地触动产品设计.

\section{2 基于可拓语义的设计方法}

可拓语义是在可拓学利用量化方法探讨物元、 事元和关系元三者间关系的层面上，加人图解思 维而提出的一种方法. 可拓语义基于 Osgood 等 ${ }^{[20]}$ 提出的语义差异量表法, 运用数据统计的方法帮 助人们探讨某一事物多重语义间的关系, 及语义 与具体图形的关联后的被接受程度, 被认为是沟 通自然科学和社会科学的桥梁 ${ }^{[21]}$; 在产品设计领 域, Lee ${ }^{[22]}$ 采用语义差异量表法对颜色在不同产品 上的情感映射进行研究，优化颜色与产品契合度; Kato 等 ${ }^{[23]}$ 运用可拓理论分析不同音色的情感，进 
行声音产品设计；段金娟等 ${ }^{[24-25]}$ 基于可拓语义对 非遗衍生产品、服务产品的设计进行研究, 验证可 拓理论在非遗衍生产品和服务产品设计上运用的 可行性; 陈香等 ${ }^{[26]}$ 基于可拓语义对智能产品的造 型设计进行研究，探讨运用可拓语义产品设计的 方法将文化特征转化为文化因子以优化智能产品 造型的可能性; 李伟丽等 ${ }^{[27]}$ 融合可拓语义和模糊 综合评价对地铁列车的外观设计进行研究, 验证 了将地域文化元素以符号的形式融人地铁列车外观 设计的方法. 这些研究都运用了可拓理论优化设计 方法解决传统设计方法中的描述信息不全、隐性知 识的存在、知识间的矛盾 ${ }^{[28-30]}$, 以及利用图解思维 表达隐性知识 ${ }^{[3]}$ 等问题. 但是, 基于民族图案基元 的可拓语义进行产品设计的研究较少.
本文从侗族织锦的图案基元出发，提出基于 民族图案基元可拓语义的产品设计方法，其流程 如图 1 所示. (1) 对侗族织锦的图案形态和语义进 行提取，在文化持有者的评估下选出最具代表性 的图案基元；（2）对该基元进行可拓语义的分析， 包括图案基元特征词汇的文化关联度评估、特征词 汇的设计文化价值评估和特征词汇的可拓设计区 间评估，以优选出可拓区间较大的特征词汇; (3) 对特征词汇的语义进行图解, 并对图解的辨识度 和设计文化价值进行评估, 得出单特征图解语义 值和图解语义的可拓区间, 以优选出可供产品设 计参考的图解；(4) 对优选出的图解进行分析，以 启发产品设计；(5) 对不同设计方案的优度进行测 评，选择优度较高的设计方案制作实物。

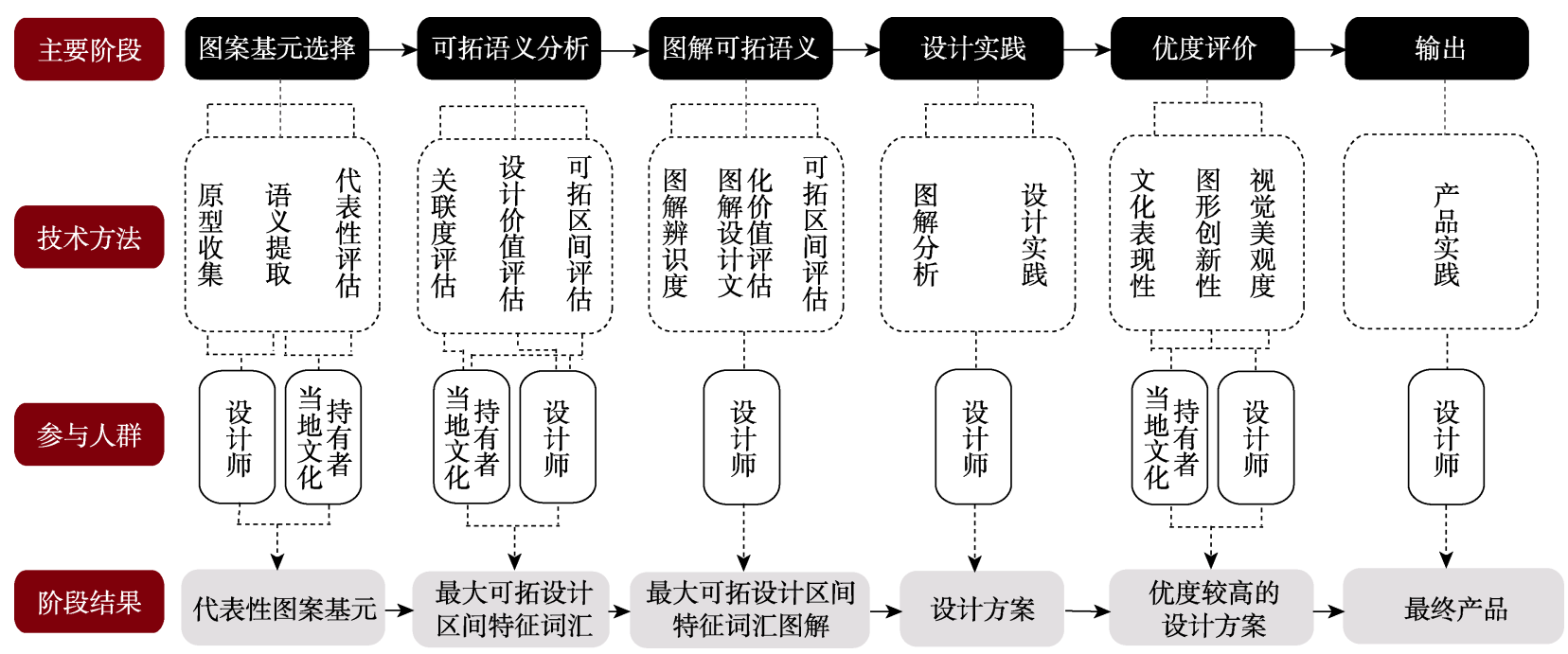

图 1 本文方法流程

\section{3 可拓语义的具体算法}

\subsection{1 图案基元的选择}

一个民族拥有多个图案基元，要进行设计实 践, 首先需要选择出最具代表性的图案基元. 设图 案基元为 $O$, 设共收集到 $m$ 个图案基元，邀请 $n$ 个 当地文化持有者对所收集到的图案基元的代表性 进行打分, 第 $i$ 个人对 $j$ 个纹样所打分值为 $a_{i j}$, 得 到第 $j$ 个纹样分值的平均值为

$$
O_{j}^{\prime}=\sum_{i=1}^{n} a_{i j} / n, i \in[1, n], j \in[1, m]
$$

通过对比不同图案基元所得到的平均值, 可 得出最具代表性的图案基元.

\subsection{2 图案基元的可拓语义分析}

根据可拓理论, 元素内涵 $P$ 以 $O$ 为研究对象
本体, $C$ 为特征, $V$ 为可拓区间的量值. 在针对民 族图案的设计中, 设 $P$ 为民族图案基元的文化内 涵, $O$ 为图案基元本体, $C$ 为图案基元的语义特 征词汇， $V$ 为语义特征的可拓区间. 将民族图案基 元文化内涵、本体、语义特征词汇之间的关系量化 描述为

$$
P=[O, C, V]
$$

每个图案基元会表达 $n$ 个语义特征词汇 $C$, 每个语义特征词汇 $C_{i}$ 对应一个可拓区间 $V_{i}$. 设共 有 $n$ 个语义特征词汇, 则式(2)分解为

$$
P=\left[\begin{array}{c}
O, C_{1}, V_{1} \\
C_{2}, V_{2} \\
\vdots \\
C_{n}, V_{n}
\end{array}\right]
$$


将 $V$ 进一步分解为 $V_{x}$ 和 $V_{y}$. 其中, $V_{x}$ 表示该特征 词汇与民族图案基元本体 $O$ 的联系程度, 即文化关 联度, $V_{x} \in(0,1], V_{x_{1}}+V_{x_{2}}+\cdots+V_{x_{n}}=1(n \geqslant 1) . V_{x}$ 越接近 1 , 该语义与基元本体 $O$ 的联系度越高. 设 每个词汇的评分区间段为 $[1, \alpha]$, 参与评估的人数 为 $\beta$, 某图案基元的某语义所得分数 $a$ 的个数为 $d_{a}$, 那么 $V_{x}$ 均值的表达式为

$$
\overline{V_{x}}=\frac{\sum_{a=1}^{\alpha} d_{a n}}{\beta \frac{\alpha(\alpha+1)}{2}}, a \in[1, \alpha], d_{a} \in[1, \beta]
$$

$V_{y}$ 表示该特征词汇在设计中的文化价值，简 称为设计价值, 数值越大, 表示该语义的设计价值 越重要. 设每个语义的设计价值重要程度评分段 为 $[1, \theta]$, 参与评分的人数为 $[1, \varphi]$, 第 $n$ 号志愿者 对某语义的评分为 $b_{n}$, 那么 $V_{y}$ 均值的表达式为

$$
\overline{V_{y}}=\frac{\sum_{n=1}^{\varphi} b_{n}}{\varphi}, b_{n} \in[1, \theta], n \in[1, \varphi]
$$

民族图案基元语义特征词汇的可拓区间为

$$
V=\overline{V_{x}} \overline{V_{y}}
$$

$V$ 值越大，该语义与图案基元的关联度越高，同时 在设计运用中的价值也越高.

\subsection{3 可拓语义的图解}

对民族图案基元的不同语义特征词汇进行关 联性的图形的图解，以 $h$ 表示，设图解的可拓区间 为 $V_{h}$; 同理, 进一步将 $V_{h}$ 分解为 $V_{h_{x}}$ 和 $V_{h_{y}} . V_{h_{x}}$ 表 示该图解的辨识度, $V_{h_{y}}$ 表示该图解的设计价值, $V_{h}$ 的表达式为

$$
V_{h}=\overline{V_{h_{x}}} \overline{V_{h_{y}}}
$$

$V_{h}$ 值越大，表示该图解的辨识度越高，同时在设 计中越能体现文化价值.

\subsection{4 优度评价}

为遴选出优度更高的设计方案, 设评价值为 $S$. 优度评价分为文化表现性 $S_{x}$ 、图案创新性 $S_{y}$ 和视觉美观度 $S_{z}$ 这 3 个方面，它们的评分值区间 均为 $[1, g]$, 且均由 $n$ 个人对其进行评分, 第 $e$ 个人 对 $f$ 个设计方案所打分值为 $a_{e f}$. 首先计算出每个 待评价对象在 3 个方面分别的平均分值为

$$
\overline{S_{x}}=\frac{\sum_{e=1}^{n} a_{e f}}{n}, e \in[1, n], f \in[1, g]
$$

$S_{y}$ 和 $S_{z}$ 均值的计算方法同式(8). 最终, 优度评分 为三者的平均值，表达式为

$$
S=\frac{\overline{S_{x}}+\overline{S_{y}}+\overline{S_{z}}}{3}
$$

\section{2 实例及结果分析}

\section{1 侗锦图案基元的选取}

侗族主要聚居于我国湘、桂、黔 3 省交界的大 山之中, 最早可以追溯到 2000 多年前的春秋战国 时期 ${ }^{[32]}$. 在长期的劳动和生活中, 侗族织锦的织 造方式、图案外观及图案语义都形成了较为稳定的 状态. 本文调研了 73 块侗族织锦, 梳理了 4 个类型 的图案基元及相关语义, 并邀请 32 名湖南省通道 县文坡村的侗族居民基于所收集的图案基元进行 评分, 主要针对单一图案对当地文化的代表程度, 分值设 1 5. 根据式(1)计算出如表 1 所示各图案基 元的代表性分值 $\overline{O_{j}^{\prime}}$, 并选择其中代表性分值 $\overline{O_{j}^{\prime}}$ 最高的风鸟纹为主要设计对象.

\section{2 侗锦图案基元的可拓语义分析}

在田野调查中，当地文化持有者对风鸟纹的 语义总结包括尊贵、神秘、灵动、美丽和圆满 5 个特征词汇，每个特征词汇相对应的可拓区间 $V$, 根据式(3)可表达为

$P=$ 侗锦代表性图案基元 $=\left[\begin{array}{r}\text { 凤鸟纹, 尊贵, } V_{1} \\ \text { 神秘, } V_{2} \\ \text { 灵动, } V_{3} \\ \text { 美丽, } V_{4} \\ \text { 圆满, } V_{5}\end{array}\right]$

针对每个特征词汇，依旧由 32 名当地文化持 有者进行打分, 按照其与凤鸟纹的文化内涵关联 的强弱程度依次计分, 关联非常紧密记 5 分, 较紧 密关联记 4 分, 基本关联记 3 分, 较弱关联记 2 分, 最弱关联记 1 分. 32 份调查问卷结果以及根据式(4) 计算出的每个特征词汇与图案基元的文化内涵关 联程度均值 $\overline{V_{x}}$ ，如表 2 所示.

另外，由 5 位长期从事侗族织锦相关产品设计 与研究的设计人员评估特征词汇的设计文化价值, 设计人员编号为 $b_{1} \sim b_{5}$, 分值设 1 5 分, 评分越高, 证明该词汇的设计价值越高. 评分结果及根据式 (5)计算出的特征词汇设计文化价值均值 $\overline{V_{y}}$ 如表 3 所示. 
表 1 侗锦图案基元的选取

\begin{tabular}{|c|c|c|c|c|c|}
\hline 基元分类 & 名称 & 造型 & 基元语义 & 特征词汇提取 & $\overline{O_{j}^{\prime}}$ \\
\hline \multirow{4}{*}{ 动物纹 } & 蜘蛛纹 & & 被视为侗族小孩的保护神，常用于婴儿背带、背面等 & $\begin{array}{l}\text { 健康、智慧、成长、 } \\
\text { 保护、坚强 }\end{array}$ & 3.72 \\
\hline & 鱼纹 & & $\begin{array}{l}\text { 侗族逐水而居, 鱼是其喜爱的食物, 并常与龙纹并用, 取鱼跃 } \\
\text { 龙门之意, 常出现于服饰之中 }\end{array}$ & $\begin{array}{l}\text { 丰收、灵动、快速、 } \\
\text { 成长、幸福 }\end{array}$ & 3.84 \\
\hline & 鸟纹 & & 相传侗族南迁时曾受到神鸟的指引，所以鸟是好运的象征 & $\begin{array}{l}\text { 好运、喜庆、灵动、 } \\
\text { 自由、飞翔 }\end{array}$ & 2.72 \\
\hline & 马纹 & & $\begin{array}{l}\text { 侗族认为其是古越人的后裔, 侗族战士善于骑马, 马纹常出 } \\
\text { 现于连绵不绝的山纹之间, 用于男性服饰居多 }\end{array}$ & $\begin{array}{l}\text { 勇猛、善战、快速、 } \\
\text { 强悍、保护 }\end{array}$ & 2.10 \\
\hline \multirow{2}{*}{ 植物纹 } & 八角花纹 & & $\begin{array}{l}\text { 侗族将所有的花都抽象为八角花, 所以八角花纹也称为百花 } \\
\text { 纹, 常用于服饰、被裖、婚庆用品 }\end{array}$ & $\begin{array}{l}\text { 美丽、幸福、繁盛、 } \\
\text { 希望、多彩 }\end{array}$ & 2.32 \\
\hline & 树纹 & & $\begin{array}{l}\text { 树纹又称为杉树纹，是侗族用来制造房屋的材料，常用于男 } \\
\text { 子服饰、鞋帽 }\end{array}$ & $\begin{array}{l}\text { 可靠、坚硬、神秘、 } \\
\text { 粗壮、丰收 }\end{array}$ & 2.55 \\
\hline \multirow{2}{*}{ 生产生活纹 } & 耕田纹 & & $\begin{array}{l}\text { 耕田纹抽象于耕地方形的外轮廓, 多与连绵的山纹相结合, } \\
\text { 再现侗族农田的实地景象, 多用于绑腿、服装边饰 }\end{array}$ & $\begin{array}{l}\text { 丰收、富裕、勤劳、 } \\
\text { 劳作、风景 }\end{array}$ & 2.43 \\
\hline & 人纹 & & $\begin{array}{l}\text { 又称为多耶纹, 来自侗族人民手拉手围火而舞的场景, 出现 } \\
\text { 在头巾、被罩、服装等多种品类中 }\end{array}$ & $\begin{array}{l}\text { 团结、友好、美满、 } \\
\text { 庆祝、跳舞 }\end{array}$ & 3.86 \\
\hline \multirow{3}{*}{ 象征再造纹 } & 太阳纹 & & $\begin{array}{l}\text { 侗族人民崇日, 并将太阳与日常所见的花朵结合起来, 形成 } \\
\text { 造型丰富的太阳纹, 多用于婴儿背带、服饰 }\end{array}$ & $\begin{array}{l}\text { 阳光、希望、热烈、 } \\
\text { 繁荣、美好 }\end{array}$ & 2.84 \\
\hline & 龙纹 & Wan & $\begin{array}{l}\text { 侗族的龙纹被抽象为起伏状的折线, 似龙游走的身体, 多用 } \\
\text { 于男性服饰, 以表尊贵 }\end{array}$ & $\begin{array}{l}\text { 尊贵、神力、权威、 } \\
\text { 神秘、成功 }\end{array}$ & 3.02 \\
\hline & 凤鸟纹 & & $\begin{array}{l}\text { 又称为凤纹, 包含鸟纹的含义, 常见的有 } 4 \text { 个凤纹相对出现, } \\
\text { 寓意八方报喜; 同时是尊贵女性的象征, 在婚庆用品中, 常与 } \\
\text { 龙纹结合运用, 成对出现 }\end{array}$ & $\begin{array}{l}\text { 尊贵、神秘、灵动、 } \\
\text { 美丽、圆满 }\end{array}$ & 3.88 \\
\hline
\end{tabular}

根据式(6)可得出如表 4 所示各特征词汇可拓 区间 $V$ 值. 可以看出，凤鸟纹特征词汇的设计可 拓区间排序为圆满、尊贵、神秘、灵动和美丽, 其 中，圆满的设计可拓区间最大，其次是尊贵、神秘， 而灵动、美丽的设计可拓区间较小. 所以，圆满作 为此次产品设计主要考虑的语义方向. 即

$$
\begin{array}{r}
P=\text { 侗锦代表性图案基元 }= \\
{\left[\begin{array}{r}
\text { 凤鸟纹, 尊贵, } 0.904 \\
\text { 神秘, } 0.713 \\
\text { 灵动, } 0.474 \\
\text { 美丽, } 0.280 \\
\text { 圆满, } 1.219
\end{array}\right]}
\end{array}
$$

表 2 特征词汇文化内涵关联度评估

\begin{tabular}{rrrrrrr}
\hline $\begin{array}{c}\text { 特征 } \\
\text { 词汇 }\end{array}$ & \multicolumn{7}{c}{ 分数 } & \multicolumn{1}{c}{$\overline{V_{x}}$} \\
\cline { 2 - 6 } 分 & 4 分 & 3 分 & 2 分 & 1 分 & \\
\hline 尊贵 & 11 & 6 & 8 & 4 & 3 & 0.238 \\
神秘 & 5 & 6 & 9 & 7 & 5 & 0.198 \\
灵动 & 0 & 5 & 7 & 10 & 10 & 0.148 \\
美丽 & 2 & 3 & 5 & 8 & 14 & 0.140 \\
圆满 & 14 & 12 & 3 & 3 & 0 & 0.277 \\
\hline
\end{tabular}

表 3 不同特征词汇设计文化价值评估分数

\begin{tabular}{ccccccc}
\hline 特征 & \multicolumn{5}{c}{ 人员编号 } & \multicolumn{1}{c}{$\overline{V_{y}}$} \\
\cline { 2 - 6 } 词汇 & $b_{1}$ & $b_{2}$ & $b_{3}$ & $b_{4}$ & $b_{5}$ & \\
\hline 尊贵 & 4 & 4 & 4 & 4 & 3 & 3.800 \\
神秘 & 2 & 4 & 4 & 3 & 5 & 3.600 \\
灵动 & 2 & 4 & 3 & 3 & 4 & 3.200 \\
美丽 & 4 & 2 & 1 & 1 & 2 & 2.200 \\
圆满 & 5 & 5 & 2 & 5 & 5 & 4.400 \\
\hline
\end{tabular}

表 4 特征词汇可拓设计区间

\begin{tabular}{cccccc}
\hline 可拓区间 & 尊贵 & 神秘 & 灵动 & 美丽 & 圆满 \\
\hline$\overline{V_{x}}$ & 0.238 & 0.198 & 0.148 & 0.140 & 0.277 \\
$\overline{V_{y}}$ & 3.800 & 3.600 & 3.200 & 2.000 & 4.400 \\
$V$ & 0.904 & 0.713 & 0.474 & 0.280 & 1.219 \\
\hline
\end{tabular}

\section{3 侗锦图案基元的可拓语义图解}

相比词语的模糊性，图形对产品设计的启发 和影响更加直接, 为了寻找与语义更为契合的图 解, 本文先邀请 5 位设计师针对每个语义选择 8 个 相关图形, 再邀请 5 位设计师与 5 位当地文化持有 者对图形进行投票, 针对每个语义选择得票率最 高的 3 个图解进行可拓性的评估, 图解的编号分别 为 $h_{1} \sim h_{3}$, 如表 5 所示. 
表 5 各特征词汇语义的图解

特征词汇

另外，邀请 3 名设计师对每个图解的辨识度 $V_{h_{x}}$ 和设计文化价值 $V_{h_{y}}$ 进行评分, 设计师编号分 别为 $W_{1} \sim W_{3}$, 相应评分如表 6 和表 7 所示.

表 6 不同设计师对各特征词汇图解辨识度的评估

\begin{tabular}{ccccccc}
\hline $\begin{array}{c}\text { 设计师 } \\
\text { 编号 }\end{array}$ & $\begin{array}{c}\text { 图解 } \\
\text { 编号 }\end{array}$ & 尊贵 & 神秘 & 灵动 & 美丽 & 圆满 \\
\hline \multirow{4}{*}{$W_{1}$} & $h_{1}$ & 0.8 & 0.7 & 0.4 & 0.6 & 0.9 \\
& $h_{2}$ & 0.5 & 0.5 & 0.5 & 0.3 & 0.2 \\
& $h_{3}$ & 0.2 & 0.4 & 0.2 & 0.3 & 0.5 \\
\hline \multirow{3}{*}{$W_{2}$} & $h_{1}$ & 0.6 & 0.5 & 0.5 & 0.4 & 0.6 \\
& $h_{2}$ & 0.3 & 0.4 & 0.3 & 0.2 & 0.1 \\
& $h_{3}$ & 0.1 & 0.2 & 0.3 & 0.1 & 0.3 \\
\hline \multirow{3}{*}{$W_{3}$} & $h_{1}$ & 0.5 & 0.4 & 0.3 & 0.5 & 0.5 \\
& $h_{2}$ & 0.2 & 0.1 & 0.1 & 0.3 & 0.1 \\
& $h_{3}$ & 0.1 & 0.1 & 0.1 & 0.1 & 0.3 \\
\hline
\end{tabular}

表 7 不同设计师对各特征词汇图解 设计文化价值的评估

\begin{tabular}{ccccccc}
\hline $\begin{array}{c}\text { 设计师 } \\
\text { 编号 }\end{array}$ & $\begin{array}{c}\text { 图解 } \\
\text { 编号 }\end{array}$ & 尊贵 & 神秘 & 灵动 & 美丽 & 圆满 \\
\hline \multirow{3}{*}{$W_{1}$} & $h_{1}$ & 5 & 2 & 3 & 4 & 5 \\
& $h_{2}$ & 3 & 4 & 2 & 3 & 2 \\
& $h_{3}$ & 2 & 1 & 2 & 3 & 3 \\
\hline \multirow{3}{*}{$W_{2}$} & $h_{1}$ & 3 & 1 & 2 & 2 & 4 \\
& $h_{2}$ & 1 & 3 & 2 & 2 & 2 \\
& $h_{3}$ & 2 & 3 & 2 & 1 & 3 \\
\hline \multirow{3}{*}{$W_{3}$} & $h_{1}$ & 4 & 2 & 3 & 3 & 4 \\
& $h_{2}$ & 1 & 3 & 1 & 2 & 3 \\
& $h_{3}$ & 1 & 2 & 2 & 2 & 3 \\
\hline
\end{tabular}

根据上述评估结果, 可以得出每个图解的辨识 度均值 $\overline{V_{h_{x}}}$ 和设计文化价值均值 $\overline{V_{h_{y}}}$, 如表 8 所示.
表 8 各特征词汇图解语义的评估均值

\begin{tabular}{ccccccc}
\hline $\begin{array}{c}\text { 图解 } \\
\text { 编号 }\end{array}$ & 均值 & 尊贵 & 神秘 & 灵动 & 美丽 & 圆满 \\
\hline$h_{1}$ & $\overline{V_{h_{x}}}$ & 0.63 & 0.53 & 0.40 & 0.50 & 0.67 \\
& $\overline{V_{h_{y}}}$ & 4.00 & 1.67 & 2.67 & 3.00 & 4.33 \\
\hline \multirow{2}{*}{$h_{2}$} & $\overline{V_{h_{x}}}$ & 0.33 & 0.33 & 0.27 & 0.26 & 0.13 \\
& $\overline{V_{h_{y}}}$ & 1.67 & 3.33 & 1.67 & 2.33 & 2.33 \\
\hline \multirow{2}{*}{$h_{3}$} & $\overline{V_{h_{x}}}$ & 0.16 & 0.23 & 0.20 & 0.17 & 0.37 \\
& $\overline{V_{h_{y}}}$ & 1.67 & 2.00 & 2.00 & 2.00 & 3.00 \\
\hline
\end{tabular}

根据式(7)，可得出每个图解的可拓语义区间， 如表 9 所示.

表 9 各特征词汇图解语义的可拓区间

\begin{tabular}{cccccc}
\hline $\begin{array}{c}\text { 图解 } \\
\text { 编号 }\end{array}$ & 尊贵 & 神秘 & 灵动 & 美丽 & 圆满 \\
\hline$h_{1}$ & 2.52 & 0.89 & 1.07 & 1.50 & 2.90 \\
$h_{2}$ & 0.55 & 1.10 & 0.45 & 0.61 & 0.30 \\
$h_{3}$ & 0.27 & 0.46 & 0.40 & 0.34 & 1.11 \\
\hline
\end{tabular}

根据风鸟纹的特征语义词汇可拓区间，在圆 满、尊贵和神秘 3 个可拓区间较大的词汇中, 可拓 区间较大的是圆满的图解 $h_{1}$. 所以, 此图解将作 为设计的主要参考元素.

\section{4 实 例}

综上所述，本文采取最具代表性的图案基元 凤鸟纹，其中语义可拓区间最大的特征词汇是圆 满, 并以圆满的可拓区间最大的圆满图解 $h_{1}$ 为参 考进行设计. 对圆满图解 $h_{1}$ 的整体外轮廓、主体图 案分布规律和辅助图形的分布规律进行提取与分 析. 该图解的外轮廓为圆形, 主体图案的构成形式 为上下、左右对称的均衡式, 内部辅助图形的排列 较为饱满且均衡, 这些均成为此次设计的参考方 向, 如图 2 所示.

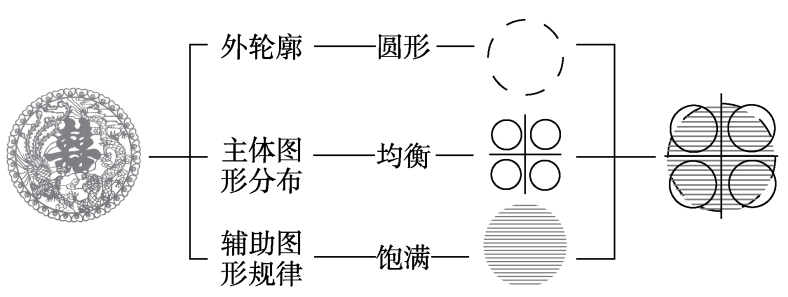

图 2 对凤鸟纹圆满图解 $h_{1}$ 的设计分析

在设计过程中, 3 个设计方案均采取类似圆形 的外轮廓, 主体图形均为风鸟纹, 设计方案 $S_{1}$ 提 取风鸟纹内部飞鸟的形态, 设计方案 $S_{2}$ 和 $S_{3}$ 均保 持凤鸟纹原始的菱形状态. 在主体图形的排列上, 3 个设计方案都基本遵循了主体图形上下、左右均 
衡分布的规律. 在中心部分, 3 个方案主体图形的 分布都借鉴了原始侗锦中凤鸟纹鸟头朝内、四面相 对的排列规律, 但设计方案 $S_{1}$ 和 $S_{3}$ 的外轮廓上设 计有风鸟纹的鸟头朝外的排列形式. 在辅助图形 上, 3 个设计方案均采用花卉纹、水波纹、折线纹 等作为填充对象，排列较为饱满且均衡，其中，设 计方案 $S_{1}$ 的辅助图形来自对其他古典图案的借鉴, $S_{2}$ 的辅助图形来自侗锦纹样, $S_{3}$ 的辅助图形来自 设计师的自我创作，如图 3 所示.

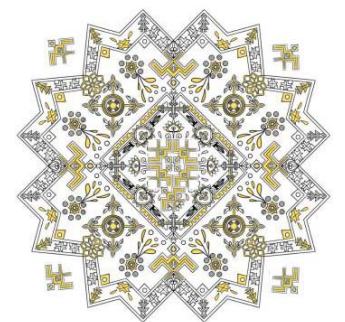

a. 设计方案 $S_{1}$

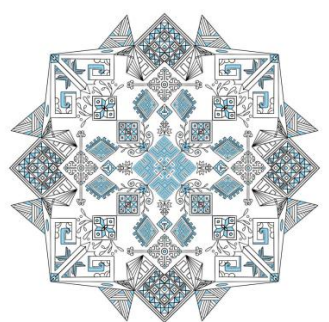

b. 设计方案 $S_{2}$

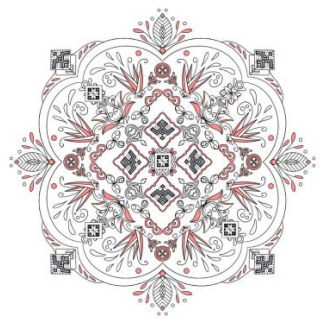

c. 设计方案 $S_{3}$

图 3 参考风鸟纹可拓语义的设计方案

\section{5 设计方案优度评价}

为了更准确地选择既能适应当地文化又能切 合现代设计需求的方案，对设计方案进行优度评 价. 设评价值为 $S$, 优度评价分为文化表现性 $S_{x}$ 、 图案创新性 $S_{y}$ 和视觉美观度 $S_{z}$ 这 3 个方面, 它们 的评分分值为 1 5. 本文共邀请了 77 位志愿者对 3 个设计方案进行网络评分, 其中, 有 38 位是设计 从业人员, 39 位是当地文化持有者. 详细问卷及评 分结果见 https://www.wjx.cn/report/108860784.aspx. 根据评分结果，用式(8)(9)计算出各个设计方案的 优度评价, 如表 10 所示. 可以看出, 设计方案 $S_{2}$ 在文化表现性、创新性及美观度方面均得到当地文 化持有者和设计从业人员的肯定; 而双方评分意 见的趋同性也证明，基于民族图案基元可拓语义 的产品设计方法能辅助提升在产品设计的过程中 双方对图案语义的理解和在视觉认同上的一致性. 所以，选择设计方案 $S_{2}$ 作为产品实践的方向，并 以此拓展设计灯具、丝巾及餐具产品. 设计效果图
及实物如图 4 所示.

表 10 不同设计方案优度评价对比

\begin{tabular}{ccccc}
\hline 设计方案编号 & $\overline{S_{x}}$ & $\overline{S_{y}}$ & $\overline{S_{z}}$ & $S$ \\
\hline$S_{1}$ & 3.74 & 3.84 & 3.90 & 3.83 \\
$S_{2}$ & 4.03 & 4.19 & 4.18 & 4.13 \\
$S_{3}$ & 3.78 & 3.97 & 3.99 & 3.91 \\
\hline
\end{tabular}

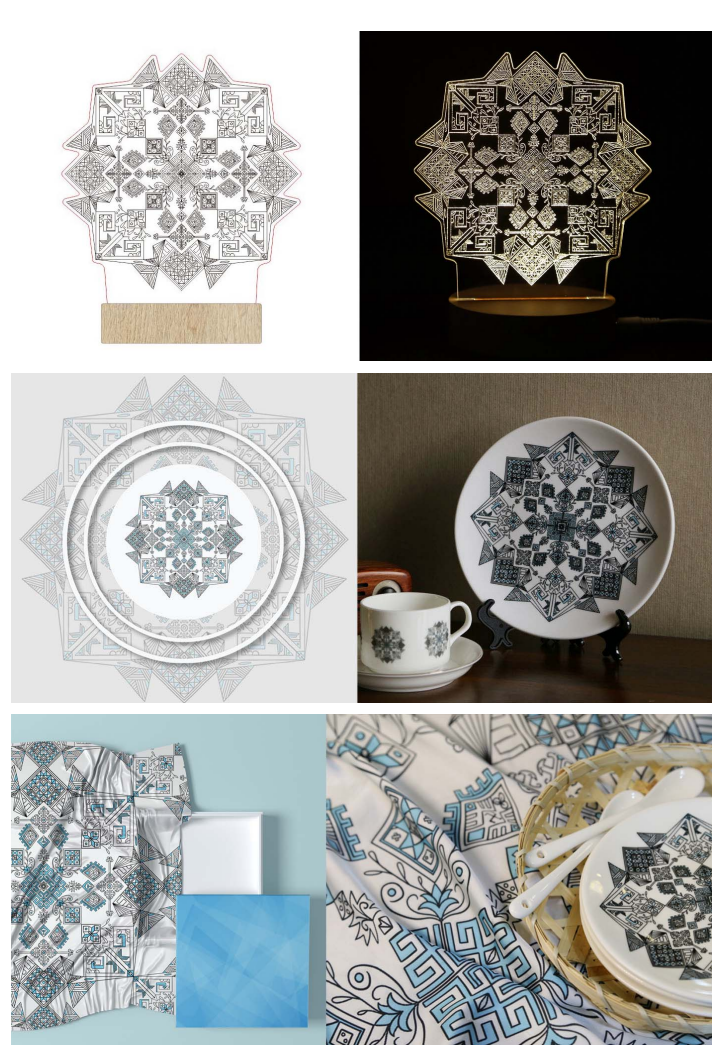

图 4 最终实践产品的设计效果图及实物

\section{3 结 语}

面向民族文化的产品设计，如何做到既兼顾 产品的创新性，又保持民族特色与文化内涵，一直 是产品设计中的重要课题. 在运用具有复杂且隐 性的图案语义的民族图案进行产品设计时, 运用 可拓理论可以提供一定的量化数据. 本文方法首 先由设计师和当地文化持有者共同提取和分析出 最具代表性的图案基元及其核心语义，作为设计 创新的基础; 其次对这些语义进行图解, 提高设计 过程中由语义到图像转化的有效率, 为设计创新 提供更好的参考; 最后根据优度评价, 选择当地文 化持有者和设计师都认同的设计作品进行产品实 践, 以保证民族图案产品设计在内涵上和视觉上 的准确性与优度. 


\section{参考文献(References):}

[1] UNESCO. Ethical principles for safeguarding intangible cultural heritage[OL]. [2020-11-03]. http://en.crihap.cn/2016-10/ 31/content 27228555.htm

[2] Zhao Haiying, Zhang Junhui. Xinjiang folk art patterns digital technology[J]. Computer Systems \& Applications, 2011, 20 (12): 167-172(in Chinese)

(赵海英, 张俊慧. 新疆民间艺术图案数字化技术 $[\mathrm{J}]$. 计算 机系统应用, 2011, 20(12): 167-172)

[3] Wu Yun. The digital construction of the basic elements of Xinjiang ethnic costumes and applied to the design[J]. Art \& Design, 2017(2): 85-87(in Chinese)

(乌云. 新疆民族服饰图案基元的数字化构建与设计运用 [J]. 装饰, 2017(2): 85-87)

[4] Liu Feiduo. Research and application of ethnic pattern primitive segmentation algorithm[D]. Beijing: Beijing University of Posts and Telecommunications, 2016(in Chinese)

(刘菲朵. 民族图案基元分割算法研究与应用 $[\mathrm{D}]$. 北京: 北 京邮电大学, 2016)

[5] Saito K, Nakamura T, Kamezawa K, et al. Japanese patterns as NFC antennas for interactive[C] //Proceedings of the 14th International Conference on Tangible, Embedded, and Embodied Interaction. New York: ACM Press, 2020: 443-451

[6] Qian J, Xiao A M, Xin X Y, et al. Pattern primitive library construction and feature analysis of Kirgiz textile pattern[J]. Humanities and Social Sciences, 2019, 7(6): 191-196

[7] Huang H, Zhang Y L, Zhai Y M. Analysis of ceramic pattern case[C] //Proceedings of International Conference on Computer-Aided Material and Engineering. Zurich: Trans Tech Publications, 2011: 41-45

[8] Dong Fuyi. Individual characteristics retrieval and applications of traditional plant carpet patterns[J]. Art \& Design, 2015(12): 124-125(in Chinese)

(董馥伊. 传统栽线毯单独纹样的特征检索与应用 [J]. 装饰, 2015(12): 124-125)

[9] Jimoh K O, Ọdẹjọ̣bí Ọ À, Fọlárànmí S A, et al. Handmade embroidery pattern recognition: a new validated database[J]. Malaysian Journal of Computing, 2020, 5(1): 390-402

[10] Sugita K, Ishida T, Miyakawa A, et al. Kansei retrieval method based on design pattern of traditional Japanese crafting object[C] //Proceedings of the 15th International Conference on Database and Expert Systems Applications. Los Alamitos: IEEE Computer Society Press, 2004: 308-312

[11] Siregar A C, Octariadi B C. Classification of sambas traditional fabric "Kain Lunggi" using texture feature[J]. Indonesian Journal of Computing and Cybernetics Systems, 2019, 13(4): 389-398

[12] Rangkuti A H, Harjoko A, Putro A E. Content based batik im- age retrieval[J]. Journal of Computer Science, 2014, 10(6): 925-934

[13] Rangkuti A H, Rasjid Z E, Santoso D. Batik image classification using treeval and treefit as decision tree function in optimizing content based batik image retrieval[J]. Procedia Computer Science, 2015, 59: 577-583

[14] Jia Xiaojun, Deng Hongtao, Liu Zihao, et al. Vein pattern classification based on VGGNet convolutional neural network for blue calico[J]. Journal of Optoelectronics Laser, 2019, 30(8): 867-875(in Chinese)

(贾小军, 邓洪涛, 刘子豪, 等. 基于 VGGNet 卷积神经网络 的蓝印花布纹样分类[J]. 光电子·激光, 2019, 30(8): 867-875)

[15] Jia Xiaojun, Ye Lihua, Deng Hongtao, et al. Elements classification of vein patterns using convolutional neural networks for blue calico[J]. Journal of Textile Research, 2020, 41(1): 110-117(in Chinese)

(贾小军, 叶利华, 邓洪涛, 等. 基于卷积神经网络的蓝印花 布纹样基元分类 [J]. 纺织学报, 2020, 41(1): 110-117)

[16] He Xuan. Repeat pattern primitive segmentation and retrieval algorithm research of printed fabric[D]. Hangzhou: Zhejiang Sci-Tech University, 2019(in Chinese) (何旋. 印花织物循环图案基元分割与检索算法研究 $[D]$. 杭 州: 浙江理工大学, 2019)

[17] Goldschmidt G, Sever A L. Inspiring design ideas with texts[J]. Design Studies, 2011, 32(2): 139-155

[18] Polanyi M. Personal knowledge: towards a post-critical philosopy[M]. London: Routledge \& Kegan Paul Ltd, 1962: 617-618

[19] Dai Rui. Product form design semantics and communication [M]. Beijing: Higher Education Press, 2010: 67-69(in Chinese) (戴瑞. 产品形态设计语义与传达 $[\mathrm{M}]$. 北京: 高等教育出版 社, 2010: 67-69)

[20] Osgood C E, Suci G J, Tannenbraum P H. The measurement of meaning[M]. Chicago: University of Illinois Press, 1957

[21] Li Youping. Extenics: a bridge between natural science and social science[J]. Science \& Technology Review, 2014, 32(36): 1 (in Chinese)

(李幼平. 可拓学: 沟通自然科学与社会科学的桥梁 $[\mathrm{J}]$. 科 技导报, 2014, 32(36): 1)

[22] Lee T R. A new comparison of psychological meaning of colors in samples and objects with semantic ratings[C] //Proceedings of the 9th Congress of the International Colour Association. Bellingham: Society of Photo-Optical Instrumentation Engineers, 2002: 418-421

[23] Kato Y, Saeki T. A new association analysis method for semantic differential data and its application to tone color analysis[C] //Proceedings of the IEEE International Conference on System, Man, and Cybernetic. Los Alamitos: IEEE Computer Society Press, 2008: 125-130 
[24] Duan Jinjuan, Li Feixia, Zhao Yuan. Design method of intangible cultural heritage derivatives based on extension semiotics[J]. Packaging Engineering, 2020, 41(12): 93-98(in Chinese) (段金娟, 李飞霞，赵远. 基于可拓符号学的非遗衍生品设 计方法研究 [J]. 包装工程, 2020, 41(12): 93-98)

[25] Duan Jinjuan, Zhang Feng, Zhou Xin. Service product design based on intangible cultural heritage and extension semantics [J]. Journal of Graphics, 2019, 40(4): 783-789(in Chinese) (段金娟，张峰，周金金. 基于非遗和可拓语义的服务产品设 计 $[\mathrm{J}]$. 图学学报, 2019, 40(4): 783-789)

[26] Chen Xiang, Yang Rui. Intelligent speaker modeling design based on extension semantic analysis[J]. Packaging Engineering, 2020, 41(14): 168-173(in Chinese)

(陈香, 杨瑞. 基于可拓语义分析的智能音箱造型设计 $[\mathrm{J}]$. 包装工程, 2020, 41(14): 168-173)

[27] Li Weili, Xiang Zerui, Li Ran, et al. Study on the approach to exterior design for metro trains based on extension semantics and fuzzy comprehensive evaluation[J]. Journal of Graphics, 2020, 41(5): 814-823 (in Chinese)

(李伟丽, 向泽锐, 李然, 等. 融合可拓语义和模糊综合评价 的地铁列车外观设计方法 [J]. 图学学报, 2020, 41(5): 814-823)

[28] Wang Jing, Jia Chengwei, Zhang Jianpei, et al. Description logic extension based on extenics theory[J]. Journal of Computer Applications, 2008, 28(8): 2071-2073+2076(in Chinese) (王静，贾成伟，张健沛，等. 基于可拓理论的描述逻辑扩展 [J]. 计算机应用, 2008, 28(8): 2071-2073+2076)

[29] Cai W. Extension theory and its application[J]. Chinese Science Bulletin, 1999, 44(17): 1538-1548

[30] Cai Wen, Yang Chunyan. Basic theory and methodology on extenics[J]. Chinese Science Bulletin, 2013, 58(13): 1190-1199 (in Chinese)

(蔡文, 杨春燕. 可拓学的基础理论与方法体系 $[\mathrm{J}]$. 科学通 报, 2013, 58(13): 1190-1199)

[31] Luo Shijian, Zhu Shangshang, Ying Fangtian, et al. Statues and progress of research on users' tacit knowledge in product design[J]. Computer Integrated Manufacturing Systems, 2010, 16 (4): 673-688(in Chinese)

(罗仕鉴, 朱上上, 应放天, 等. 产品设计中的用户隐性知识 研究现状与进展 $[\mathrm{J}]$. 计算机集成制造系统，2010，16(4): 673-688)

[32] Zhou Yahui. Inheritance and reproduction: research on Tongdao Dong brocade in Hunan Province[M]. Beijing: Science Press, 2018(in Chinese)

(周亚辉. 传承与再生产: 湖南通道侗锦研究 $[\mathrm{M}]$. 北京: 科学出版社, 2018) 